\title{
Preface Issue 1-2012
}

\author{
Hans-Christoph Grunau
}

Published online: 9 February 2012

(C) Deutsche Mathematiker-Vereinigung and Springer Verlag 2012

In Issue 2-2010 Francisco Santos summarised in a joint survey article with Edward Kim the state of the art concerning the works on the Hirsch-conjecture from polytope geometry. During the final composition of this article, Francisco Santos found a counterexample to this conjecture and published it on the arXiv, see also Mitteilungen der DMV 18 (2010), no. 4, 214-221. For the latest annual meeting of the DMV he gave a plenary lecture on this topic and explained some of the background. While working on the survey article, as Francisco Santos mentioned, he looked up so many related works and thought so much about results indicating that the conjecture might possibly be false that finally, he got the basic idea and found his counterexample. In the subsequent discussion Günter Ziegler summarised that when one wishes to prove or disprove any important conjecture one should first write (or at least read) a corresponding survey article for Jahresbericht der DMV.

As an editor of Jahresbericht I enjoyed this very much: It becomes once more evident that survey articles also play an important role in active research, a fact which is possibly sometimes underestimated. In this spirit I would like to encourage all readers of the Jahresbericht to mention suitable topics for interesting and comprehensible future survey articles to the editors; for this one may also use the online forum https://dmv.mathematik.de/forum.html.

The first article in the current issue is also in the area of convex and discrete geometry and is devoted to "combinatorial reciprocity theorems". Characteristic polynomials of hyperplane arrangements and Ehrhart-polynomials of polytopes are examples of counting functions encoding basic geometric information. Such counting functions are usually evaluated on the positive integers. Matthias Becks's article describes some

\footnotetext{
H.-Ch. Grunau ( $\bowtie)$

Institut für Analysis und Numerik, Fakultät für Mathematik, Otto-von-Guericke-Universität, Postfach 4120, 39016 Magdeburg, Germany

e-mail: hans-christoph.grunau@ovgu.de
} 
interesting connections-i.e. reciprocity theorems-which show up when negative integers are inserted.

Ulrich Felgner's historical article on the principle of mathematical induction describes its emergence in the 16th and 17th century, its antecedents in antiquity, and its formalisation, specification and generalisation in the 19th and 20th century. Ulrich Felgner includes numerous interesting logical and philosophical considerations on this presently so frequently used method of proof.

New books on the theory of three dimensional dynamical systems and from algebraic topology are reviewed.

Since 1899, Teubner-and for the last four years Vieweg+Teubner-has been the publishing house for the "Jahresbericht der DMV". Having looked carefully at the cover of the current issue you will have observed that the logo is now different: Indeed, from now on the "Jahresbericht der DMV" is a member of the big and renowned family of the "Springer" journals. On the one hand one may regret that the brand "Teubner" has now completely disappeared. On the other hand, "Vieweg+Teubner" has been part of the Springer group, and for two years Springer has already been responsible for the technical production of our journal. Being a "Springer" journal opens a number of new opportunities, increasing the attractiveness of Jahresbericht for authors and facilitating its international dissemination. A first step in this direction was already made by making the Jahresbericht available online on Springerlink. Hoping that an increasingly international readership will take notice of the articles of Jahresbericht means that in accordance with the development of the past years, English is the preferential publication language. At the same time, articles written in German will continue to be accepted. One may regret that the "Vorwort" is no longer written in German but I am confident that the new conditions are quite advantageous for the "Jahresbericht der DMV". 